PROCEEDINGS OF THE

AMERICAN MATHEMATICAL SOCIETY

Volume 138, Number 1, January 2010, Pages 217-223

S 0002-9939(09)10000-X

Article electronically published on August 12, 2009

\title{
MINIMAL GROUP ACTIONS ON DENDRITES
}

\author{
ENHUI SHI, SUHUA WANG, AND LIZHEN ZHOU \\ (Communicated by Alexander N. Dranishnikov)
}

\begin{abstract}
Minimal group actions on dendrites appear naturally in the study of 3-dimensional hyperbolic geometry. In this paper, it is shown that if a group $G$ acts on a nondegenerate dendrite $X$ minimally, then $X$ admits no $G$-invariant measure. In particular, $G$ cannot be amenable.
\end{abstract}

\section{INTRODUCTIONS AND PRELIMINARIES}

By a Kleinian group we mean a group $\Gamma$ acting freely and properly discontinuously on hyperbolic 3 -space, $\mathbf{H}^{3}$. The limit set $\Lambda(\Gamma)$ of $\Gamma$ consists of the points $x \in \partial \mathbf{H}^{3}$ such that there is a sequence $\gamma_{i} \in \Gamma$ and some $y \in \mathbf{H}^{3}$ with $\lim _{i \rightarrow \infty} \gamma_{i}(y)=x$. Clearly $\Lambda(\Gamma)$ is a $\Gamma$-invariant closed subset of $\partial \mathbf{H}^{3}$. It is well known that the action of $\Gamma$ on $\Lambda(\Gamma)$ is minimal; that is, there is no nonempty proper $\Gamma$-invariant closed subset of $\Lambda(\Gamma)$ (see [7, page 601] and (b) of Remark 1.2 below). In some cases, $\Lambda(\Gamma)$ is known to be a dendrite, and its Hausdorff dimension has been calculated by some authors (see e.g. [2, 3]). These facts motivate us to study the minimal dynamical systems of group actions on dendrites. In fact, the topological dynamical systems of group actions on dendrites have been studied by some authors (see e.g. [4, 8] ).

Before stating our main results, let us recall some notions. Let $X$ be a topological space and let $\operatorname{Homeo}(X)$ be the homeomorphism group of $X$. Suppose that $G$ is a discrete topological group. By an action of $G$ on $X$, we mean a group homomorphism $\phi: G \rightarrow \operatorname{Homeo}(X)$. For convenience, we often use the pair $(X, G)$ to denote a group action, and we denote $\phi(g)(x)$ simply by $g x$ or $g(x)$.

The $G$-orbit of a point $x \in X$ is the set $G x=\{g x: g \in G\}$. For a subset $A \subseteq X$, let $G A=\bigcup_{x \in A} G x$. A subset $A \subseteq X$ is said to be $G$-invariant if $G A=A$. A Borel measure $\mu$ on $X$ is $G$-invariant if $\mu(g A)=\mu(A)$ for any $g \in G$ and for any Borel subset $A$ of $X$.

Definition 1.1. The action of $G$ on $X$ is

(a) topologically transitive if for any two nonempty open subsets $U$ and $V$ of $X$, there is some $g \in G$ such that $g(U) \cap V \neq \emptyset$.

(b) weakly mixing if the diagonal action of $G$ on the product space $X \times X$ is topologically transitive, or equivalently for any two families of nonempty open

Received by the editors November 23, 2008, and, in revised form, April 10, 2009, and April 14, 2009.

2000 Mathematics Subject Classification. Primary 37B05, 57M50.

Key words and phrases. Dendrite, minimal group action, amenable group, invariant measure, weak mixing.

(C)2009 American Mathematical Society Reverts to public domain 28 years from publication 
subsets $U_{1}, U_{2}$ and $V_{1}, V_{2}$ of $X$, there is some $g \in G$ such that $g\left(U_{i}\right) \cap V_{i} \neq \emptyset$ for each $i=1,2$.

(c) point transitive if there is some point $x \in X$ such that the $G$-orbit $G x$ is dense in $X$. Such an $x$ is called a transitive point.

(d) minimal if $G x$ is dense in $X$ for each $x \in X$.

Remark 1.2. For Definition 1.1, we have

(a) when $G$ is countable and $X$ is a Polish space, the two notions of topological transitivity and point transitivity are the same, and in fact the collection of transitive points form a dense $G_{\delta}$ set in $X$.

(b) the condition (d) is equivalent to saying that $X$ has no proper $G$-invariant closed subset of $X$.

A continuum is a compact connected metric space. A continuum is degenerate if it is a point. A dendrite $X$ is a locally connected continuum containing no simple closed curve.

A topological group $G$ is amenable if there is an invariant mean on $\mathcal{B}(G)$, the bounded right uniformly continuous functions on $G$. When $G$ is discrete, there is an intrinsic characterization given by Føler: $G$ is amenable if and only if for any $\epsilon>0$ and any finite subset $K$ of $G$, there is a finite set $A$ of $G$ such that $|g A \triangle A| /|A|<\epsilon$, for all $g \in K$, where || denotes a cardinal number. It is well known that every abelian group must be amenable and every amenable group action on a compact metric space must have an invariant measure. For details about amenability, one may consult [6].

In this paper, we prove the following:

Theorem 1.3. If a group $G$ acts on a nondegenerate dendrite $X$ minimally, then $X$ admits no $G$-invariant measure. In particular, $G$ cannot be amenable.

The proof of the theorem relies in the structure theory of minimal flows established by H. Furstenberg and R. Ellis et al.

\section{REgionally PROXimal RELATIONS AND WEAK MiXiNG}

Let $G$ be a discrete group, $X$ a metric space with metric $d$, and $\phi: G \rightarrow$ $\operatorname{Homeo}(X)$ a group action. Recall that $\phi$ is said to be equicontinuous if for any $\epsilon>0$ there is a $\delta>0$ such that whenever $d(x, y)<\delta$, then $d(g x, g y)<\epsilon$, for all $g \in G$.

Let $\phi: G \rightarrow \operatorname{Homeo}(X)$ and $\psi: G \rightarrow \operatorname{Homeo}(Y)$ be two actions of group $G$ on compact metric spaces. If there is a continuous surjection $\pi: X \rightarrow Y$ such that for all $x \in X$ and $g \in G, \pi(\phi(g)(x))=\psi(g)(\pi(x))$, then $(Y, G)$ is said to be a factor of $(X, G)$. It is well known that $(Y, G)$ is a factor of $(X, G)$ if and only if there is a closed $G$-invariant equivalence relation $R \subset X \times X$ such that $Y=X / R$.

Recall that the equicontinuous structure relation $R_{e q}$ is the smallest closed $G$ invariant equivalence relation $S \subset X \times X$ such that the factor $(X / S, G)$ is equicontinuous (see [1, page 126]). The regionally proximal relation $Q$ consists of the pair $(x, y) \in X \times X$ such that there are sequences $\left\{\left(x_{i}, y_{i}\right)\right\}$ in $X \times X$ and $\left\{g_{i}\right\}$ in $G$ such that $x_{i} \rightarrow x, y_{i} \rightarrow y$ and $d\left(g_{i} x_{i}, g_{i} y_{i}\right) \rightarrow 0$ as $i \rightarrow \infty$. Clearly $Q$ is closed, but it is not an equivalence relation in general. However it is really the case under some assumptions, just as the following theorem shows (see e.g. [1, page 130]). 
Theorem 2.1. Let $(X, G)$ be a minimal group action with an invariant Borel probability measure $\lambda$. Then the equicontinuous structure relation $S_{e q}$ coincides with the regionally proximal relation $Q$.

The following theorem is well known in dynamical systems (see e.g. 1, page 133]).

Theorem 2.2. Let $(X, G)$ be a minimal group action, with $X$ metric, which admits an invariant measure $\lambda$. Then the following are equivalent:

(a) $(X, G)$ is weakly mixing.

(b) $(X, G)$ has no non-trivial equicontinuous factor (i.e., $\left.S_{e q}=X \times X\right)$.

Now we list some facts about dendrites in the following lemma which will be used in the following proofs. For details one may consult [5].

Lemma 2.3. For a dendrite $X$, we have the following facts:

(a) For any two points $x, y \in X$ there is a unique arc $[x, y]$ in $X$ connecting $x$ and $y$.

(b) Every subcontinuum of $X$ is a dendrite, and every connected subset of $X$ is arcwise connected.

(c) Every point $x \in X$ has a neighborhood basis consisting of subdendrites.

The following lemma can be deduced from [5, 8.30]. For completeness, we give a direct proof here.

Lemma 2.4. Let $X$ be a dendrite with metric $d$. Then for any $\epsilon>0$ there is a $\delta=\delta(\epsilon) \in(0, \epsilon / 2]$ such that for any $x, y \in X$ with $0<d(x, y) \leqslant \delta$, the diameter $\operatorname{diam}([x, y])<\epsilon$.

Proof. Assume that the conclusion is false. Then there exist $\epsilon_{0}>0$ and $x_{i}, y_{i} \in X$ such that $d\left(x_{i}, y_{i}\right) \rightarrow 0$ as $i \rightarrow \infty$ and $\operatorname{diam}\left(\left[x_{i}, y_{i}\right]\right) \geq \epsilon_{0}$ for all $i$. Passing through a subsequence if necessary, we may suppose that $x_{i} \rightarrow z$ and $y_{i} \rightarrow z$ as $i \rightarrow \infty$, for some $z \in X$. Choose a dendrite neighborhood $U$ of $z$ with $\operatorname{diam}(U)<\epsilon_{0} / 2$. Then, for sufficiently large $i$, we have $x_{i}, y_{i} \in U$. Thus $\left[x_{i}, y_{i}\right] \subseteq U$. This implies that $\operatorname{diam}\left(\left[x_{i}, y_{i}\right]\right)<\epsilon_{0}$, which is a contradiction. Thus we complete the proof.

Let $R \subset X \times X$ be a relation on $X$ and $x \in X$. We use the symbol $R[x]$ to denote the set $\{y \in X:(x, y) \in R\}$. Then we have

Lemma 2.5. Let $(X, G)$ be a group action where $X$ is a dendrite with metric d. Then for every $x \in X, Q[x]$ is a subdendrite of $X$ where $Q$ is the regionally proximal relation for $(X, G)$.

Proof. Let $x, y$ be any two different points in $X$ with $(x, y) \in Q$. Then there are sequences $\left(x_{i}, y_{i}\right) \in X \times X$ and $g_{i} \in G$ with $x_{i} \rightarrow x, y_{i} \rightarrow y$ and $d\left(g_{i} x_{i}, g_{i} y_{i}\right) \rightarrow 0$ as $i \rightarrow \infty$. Let $[x, y]$ be the (unique) arc from $x$ to $y$. Then for every $z \in[x, y]$ with $z \neq x$ and $z \neq y$ there are dendrite neighborhoods $U_{x}$ of $x$ and $U_{y}$ of $y$ with $U_{x} \cap U_{y}=\emptyset$ and $z \notin U_{x} \cup U_{y}$ by (c) of Lemma 2.3. Thus for sufficiently large $i$, we have $x_{i} \in U_{x}$ and $y_{i} \in U_{y}$. It follows from (a) and (b) of Lemma 2.3 that the $\operatorname{arc}[x, y]$ is contained in the subcontinuum $\left[x, x_{i}\right] \cup\left[x_{i}, y_{i}\right] \cup\left[y_{i}, y\right]$. Since $\left[x, x_{i}\right] \subset U_{x}$ and $\left[y_{i}, y\right] \subset U_{y}$ by (a) and (b) of Lemma 2.3 , we have $z \in\left[x_{i}, y_{i}\right]$. Since $\operatorname{diam}\left(\left[g_{i} x_{i}, g_{i} y_{i}\right]\right) \rightarrow 0$ as $i \rightarrow \infty$ by Lemma 2.4 , we obtain that $d\left(g_{i} x_{i}, g_{i} z\right) \rightarrow 0$ as $i \rightarrow \infty$. By the definition of regionally proximal relation, we see that $(x, z) \in Q$. Since $z$ is arbitrary, we get that $[x, y] \subset Q$. This implies that $Q[x]$ is connected, and so $Q[x]$ is a subdendrite by the fact that $Q$ is a closed relation. Thus we complete the proof. 


\section{Proof of the MAIN THEOREM}

Let $X$ and $Y$ be two topological spaces. Recall that a continuous map $f: X \rightarrow Y$ is said to be monotone if $f^{-1}(y)$ is connected for each $y \in Y$.

The following theorem is well known in Continuum Theory (see [5, Corollary 13.41]).

Theorem 3.1. Let $X$ be a dendrite and $Y$ be a continuum. If $f$ is a monotone continuous map from $X$ onto $Y$, then $Y$ is a dendrite.

Recall that a continuum $X$ is homogeneous if for any two points $x, y \in X$ there is a homeomorphism $h$ of $X$ onto itself such that $h(x)=y$. A point $x$ in a continuum $X$ is called a cut point if $X-\{x\}$ is not connected and is called a non-cut point if $X-\{x\}$ is connected. By the well known non-cut point existence theorem due to R. L. Moore (see [5, page 89]), we know that a nondegenerate dendrite must have at least two non-cut points. In addition, it is known that a nondegenerate dendrite must have uncountably many cut points (see [5, page 168]). Thus a nondegenerate dendrite cannot be homogenous; that is, we have the following lemma.

Lemma 3.2. There is no nondegenerate homogenous dendrite.

The following theorem is only part of Theorem 6 in Chapter 3 of [1, page 53].

Theorem 3.3. If a compact metric space $X$ admits an equicontinuous minimal group action, then $X$ must be homogeneous.

Lemma 3.4. Let $X$ be a dendrite and $(X, G)$ be a minimal action of group $G$ with an invariant Borel probability measure $\mu$. Then $(X, G)$ must be weakly mixing.

Proof. Let $Q$ be the regionally proximal relation for $(X, G)$. From Lemma 2.5 we know that $Q[x]$ is connected for each $x \in X$. Thus the factor map $\pi: X \rightarrow$ $X / Q, x \mapsto Q[x]$ is monotone, and thus $X / Q$ is a dendrite by Theorem 3.1. From Theorem 2.1 we see that the factor system $(X / Q, G)$ is minimal and equicontinuous. It follows from Lemma 3.2 that $X / Q$ must be degenerate; that is, $X / Q$ is a point. Therefore $Q=X \times X$. So, by Theorem 2.2, $(X, G)$ is weakly mixing. Thus we complete the proof.

Let $X$ be a metric space with metric $d$ and $G$ be a discrete group. The action of $G$ on $X$ is said to be sensitive if there is some $c>0$ such that for any nonempty open subset $U$ of $X$ there is a $g \in G$ such that $\operatorname{diam}(g(U))>c$. The number $c$ is said to be a sensitive constant.

Lemma 3.5. Let $(X, G)$ be a weakly mixing group action, where $X$ is a dendrite with metric $d$. Then $(X, G)$ is sensitive.

Proof. Let $c=\operatorname{diam}(X) / 8$. Choose two points $x_{0}$ and $y_{0}$ in $X$ such that $d\left(x_{0}, y_{0}\right)=$ $4 c$. Let $B_{d}\left(x_{0}, c\right)$ and $B_{d}\left(y_{0}, c\right)$ be open balls of radius $c$ and centers $x_{0}$ and $y_{0}$ respectively. Then we have $d\left(B_{d}\left(x_{0}, c\right), B_{d}\left(y_{0}, c\right)\right)>c$. For an arbitrary nonempty open subset $U \subseteq X$, since $(X, G)$ is weakly mixing, there is some $g \in G$ such that $g(U) \cap B_{d}\left(x_{0}, c\right) \neq \emptyset$ and $g(U) \cap B_{d}\left(y_{0}, c\right) \neq \emptyset$. Then $\operatorname{diam}(g(U))>c$. Hence, $c$ is a sensitive constant for the action. Thus we complete the proof.

Before proving our main theorem, let us recall some definitions, following Mai and the first author 4 . Let $A$ be an $\operatorname{arc} \operatorname{End}(A)$ denotes the set of two endpoints of $A$, and $\stackrel{\circ}{A}=A-\operatorname{End}(A)$. 
For a dendrite $X$ and an $\operatorname{arc} A$ in $X$, define

$$
X(A)=A \cup(\bigcup\{Y: Y \text { is a component of } X-A \text {, and } \bar{Y} \cap \stackrel{\circ}{A} \neq \emptyset\}) .
$$

$X(A)$ is called a subdendrite of $X$ strung by $A$, and $A$ is called the trunk of $X(A)$ (see [4]).

The following properties can be deduced directly from the definition above.

Lemma 3.6. Let $X(A)$ be a subdendrite of $X$ strung by $A$. Then:

(a) $X(A)-\operatorname{End}(A)$ is open in $X$.

(b) If $A^{\prime}$ is a subarc of $A$, then $X\left(A^{\prime}\right) \subset X(A)$.

(c) If $f: X \rightarrow X$ is a homeomorphism, then $f(X(A))=X(f(A))$.

Let $A$ be an arc, and let $\prec$ be an ordering in $A$. If there is a homeomorphism $h: A \rightarrow[0,1]$ such that, for any $x, y \in A, x \prec y$ if and only if $h(x)<h(y)$, then $\prec$ is called a natural ordering. Suppose that $y_{0}, y_{1}, \cdots, y_{m}$ are points in the arc $A$, $m \geqslant 2$, and $\operatorname{End}(A)=\left\{y_{0}, y_{m}\right\}$. If $y_{0} \prec y_{1} \prec y_{2} \cdots \prec y_{m-1} \prec y_{m}$ via a natural ordering $\prec$ in $A$, and $d\left(y_{k}, y_{0}\right)=k \cdot d\left(y_{m}, y_{0}\right) / m$ for all $k=1, \cdots, m$, then the sequence $\left(y_{1}, y_{2}, \cdots, y_{m-1}\right)$ is said to be a set of pseudo $m$-section points of $A$ from $y_{0}$ to $y_{m}$ (see [4]).

Lemma 3.7. Let $X$ be a dendrite with metric d. Fix an $\epsilon>0$, and let $\delta=\delta(\epsilon)$ be as in Lemma 2.4. Suppose $\left[x_{0}, x_{5}\right]$ is an arc in $X$ and $\left(x_{1}, \cdots, x_{4}\right)$ is a set of pseudo 5-section points of $\left[x_{0}, x_{5}\right]$ from $x_{0}$ to $x_{5}$. Also, suppose $\left[y_{0}, y_{5}\right]$ is an arc in $X$ and $\left(y_{1}, \cdots, y_{4}\right)$ is a set of pseudo 5-section points of $\left[y_{0}, y_{5}\right]$ from $y_{0}$ to $y_{5}$, and $d\left(x_{0}, x_{5}\right)=d\left(y_{0}, y_{5}\right)=5 \epsilon$. If $d\left(x_{i}, y_{i}\right)<\delta$ for all $i=0, \cdots, 5$, then $\left[y_{2}, y_{3}\right] \subset\left[x_{1}, x_{4}\right]$.

Proof. By Lemma 2.4, we have that $\operatorname{diam}\left(\left[x_{1}, y_{1}\right]\right)<\epsilon$ and $\operatorname{diam}\left(\left[x_{4}, y_{4}\right]\right)<\epsilon$. Observe that there exists a unique point $a \in\left[x_{1}, y_{1}\right]$ such that $\left[x_{1}, a\right] \cap\left[y_{0}, y_{5}\right]=\{a\}$. (The point $a$ may be $x_{1}$ or $y_{1}$.) Also, there exists a unique point $b \in\left[x_{4}, y_{4}\right]$ such that $\left[x_{4}, b\right] \cap\left[y_{0}, y_{5}\right]=\{b\}$. Since $\operatorname{diam}\left(\left[x_{1}, a\right]\right)<\epsilon, \operatorname{diam}\left(\left[x_{4}, b\right]\right)<\epsilon$ and $d\left(x_{1}, x_{4}\right) \geqslant 3 \epsilon$, then $\left[x_{1}, a\right] \cap\left[x_{4}, b\right]=\emptyset$. Thus $\left[x_{1}, a\right] \cup[a, b] \cup\left[b, x_{4}\right]$ is an arc joining $x_{1}$ and $x_{4}$. Hence $\left[x_{1}, a\right] \cup[a, b] \cup\left[b, x_{4}\right]=\left[x_{1}, x_{4}\right]$, which implies that $[a, b] \subset\left[x_{1}, x_{4}\right]$. Since $d\left(y_{1},\left\{y_{0}, y_{2}\right\}\right) \geqslant \epsilon$ and $\operatorname{diam}\left(\left[a, y_{1}\right]\right)<\epsilon$, we get $a \in\left[y_{0}, y_{2}\right]-\left\{y_{0}, y_{2}\right\}$. Also, since $d\left(y_{4},\left\{y_{3}, y_{5}\right\}\right) \geqslant \epsilon$ and $\operatorname{diam}\left(\left[b, y_{4}\right]\right)<\epsilon$, we have $b \in\left[y_{3}, y_{5}\right]-\left\{y_{3}, y_{5}\right\}$. Therefore, $\left[y_{2}, y_{3}\right] \subset[a, b] \subset\left[x_{1}, x_{4}\right]$. Thus we complete the proof.

Now we shall give the proof of the main theorem in this paper.

Proof of Theorem 1.3. Assume to the contrary that there is a $G$-invariant Borel measure $\mu$ on $X$. Since $(X, G)$ is minimal, the support of $\mu, \operatorname{supp}(\mu)=X$.

By Lemma 3.4, $(X, G)$ is weakly mixing and hence is sensitive by Lemma 3.5. Let $c_{0}$ be a sensitive constant for the action, and let $c=c_{0} / 30$. First take two points $y_{0,0}, y_{0,5} \in X$ such that $d\left(y_{0,0}, y_{0,5}\right)=5 c$. Let $\left(y_{0,1}, \cdots, y_{0,4}\right)$ be a set of pseudo 5 -section points of $\left[y_{0,0}, y_{0,5}\right]$ from $y_{0,0}$ to $y_{0,5}$. For the subdendrite $X\left(\left[y_{0,2}, y_{0,3}\right]\right)$, since $(X, G)$ is sensitive, there is some $g_{1} \in G$ such that $\operatorname{diam}\left(g_{1}\left(X\left(\left[y_{0,2}, y_{0,3}\right]\right)\right)\right)>$ $c_{0}(=30 c)$. Let $A_{1}=g_{1}\left(\left[y_{0,2}, y_{0,3}\right]\right)$. Then $g_{1}\left(X\left(\left[y_{0,2}, y_{0,3}\right]\right)\right)=X\left(g_{1}\left(\left[y_{0,2}, y_{0,3}\right]\right)\right)=$ $X\left(A_{1}\right)$ by $(\mathrm{c})$ of Lemma 3.6.

Consider the subdendrite $X\left(A_{1}\right)$. We now choose two points $y_{1,0}, y_{1,5} \in X\left(A_{1}\right)$ such that $d\left(y_{1,0}, y_{1,5}\right)=5 c$ and $X\left(\left[y_{1,0}, y_{1,5}\right]\right) \subseteq X\left(A_{1}\right)$ as follows. If $\operatorname{diam}\left(A_{1}\right) \geqslant$ $10 c$, then we need only take $y_{1,0}, y_{1,5} \in A_{1}$ with $d\left(y_{1,0}, y_{1,5}\right)=5 c$. So $X\left(\left[y_{1,0}, y_{1,5}\right]\right)$ $\subseteq X\left(A_{1}\right)$; otherwise, $\operatorname{diam}\left(A_{1}\right)<10 c$. Then because $\operatorname{diam}\left(X\left(A_{1}\right)\right)>30 c$, by the 
triangle inequality, there must be a connected component $Y$ of $X\left(A_{1}\right)-A_{1}$ such that $\bar{Y} \cap \stackrel{\circ}{A}_{1}=\{x\}$ and $\operatorname{diam}(Y)>10 c$. So there is $y \in Y$ such that $d(x, y)>5 c$. Therefore we can take $y_{1,0}, y_{1,5} \in[x, y]$ such that $y_{1,0}=x$ and $d\left(y_{1,0}, y_{1,5}\right)=5 c$. It is not difficult to see that $X\left(\left[y_{1,0}, y_{1,5}\right]\right) \subseteq X\left(A_{1}\right)$. Let $\left(y_{1,1}, \cdots, y_{1,4}\right)$ be a set of pseudo 5 -section points of $\left[y_{1,0}, y_{1,5}\right]$ from $y_{1,0}$ to $y_{1,5}$.

Suppose that for each $i \leqslant n-1$ we get a subdendrite $X\left(\left[y_{i, 0}, y_{i, 5}\right]\right)$ such that $d\left(y_{i, 0}, y_{i, 5}\right)=5 c$, and $\left(y_{i, 1}, \cdots, y_{i, 4}\right)$ is a set of pseudo 5-section points of $\left[y_{i, 0}, y_{i, 5}\right]$ from $y_{i, 0}$ to $y_{i, 5}$. Moreover, $X\left(\left[y_{i, 0}, y_{i, 5}\right]\right) \subseteq g_{i}\left(X\left(\left[y_{i-1,2}, y_{i-1,3}\right]\right)\right)$ for some $g_{i} \in G$. Now for $i=n$, by the sensitivity of $(X, G)$, there is some $g_{n} \in G$ such that $\operatorname{diam}\left(g_{n}\left(X\left(\left[y_{n-1,2}, y_{n-1,3}\right]\right)\right)\right)>c_{0}(>15 c)$. Let $A_{n}=g_{n}\left(\left[y_{n-1,2}, y_{n-1,3}\right]\right)$. Then $g_{n}\left(X\left(\left[y_{n-1,2}, y_{n-1,3}\right]\right)\right)=X\left(A_{n}\right)$. Since $\operatorname{diam}\left(X\left(A_{n}\right)\right)>15 c$, using the same discussion as above, we can get $y_{n, 0}, y_{n, 5} \in X\left(A_{n}\right)$ such that $d\left(y_{n, 0}, y_{n, 5}\right)=5 c$ and $X\left(\left[y_{n, 0}, y_{n, 5}\right]\right) \subseteq X\left(A_{n}\right)$. Let $\left(y_{n, 1}, \cdots, y_{n, 4}\right)$ be a set of pseudo 5 -section points of $\left[y_{n, 0}, y_{n, 5}\right]$ from $y_{n, 0}$ to $y_{n, 5}$.

Continuing this process, we will get a sequence $\left\{g_{n}\right\}_{n \in \mathbb{N}} \subset G$ and a sequence of subdendrites $\left\{X\left(\left[y_{n, 0}, y_{n, 5}\right]\right)\right\}_{n \in \mathbb{N}}$ such that

(i) for each $n \in \mathbb{N}, d\left(y_{n, 0}, y_{n, 5}\right)=5 c$, and $\left(y_{n, 1}, \cdots, y_{n, 4}\right)$ is a set of pseudo 5 -section points of $\left[y_{n, 0}, y_{n, 5}\right]$ from $y_{n, 0}$ to $y_{n, 5}$.

(ii) for each $n \in \mathbb{N}, X\left(\left[y_{n, 0}, y_{n, 5}\right]\right) \subseteq g_{n}\left(X\left(\left[y_{n-1,2}, y_{n-1,3}\right]\right)\right)$.

Let $\delta=\delta(c)$ be as in Lemma 2.4. Since $X$ is compact, there are two integers $q>$ $p>0$ such that $\max \left\{d\left(y_{p, j}, y_{q, j}\right): j=0, \cdots, 5\right\}<\delta$. It follows from Lemma 3.7 that $\left[y_{p, 2}, y_{p, 3}\right] \subset\left[y_{q, 1}, y_{q, 4}\right]$. Thus by (b) of Lemma 3.6, $X\left(\left[y_{p, 2}, y_{p, 3}\right]\right) \subset X\left(\left[y_{q, 1}, y_{q, 4}\right]\right)$. Also, $X\left(\left[y_{q, 1}, y_{q, 4}\right]\right) \subset X\left(\left[y_{q, 0}, y_{q, 5}\right]\right)$. From condition (ii) above, we see that

$$
\left(g_{q} \cdots g_{p+1}\right)^{-1}\left(X\left(\left[y_{q, 0}, y_{q, 5}\right]\right)\right) \subset X\left(\left[y_{p, 2}, y_{p, 3}\right]\right) \subset X\left(\left[y_{q, 1}, y_{q, 4}\right]\right) .
$$

Let $g=\left(g_{q} \cdots g_{p+1}\right)^{-1}$. Since $\mu$ is $G$-invariant, then

$$
\mu\left(X\left(\left[y_{q, 0}, y_{q, 5}\right]\right)\right)=\mu\left(g\left(X\left(\left[y_{q, 0}, y_{q, 5}\right]\right)\right)\right) \leq \mu\left(X\left(\left[y_{q, 1}, y_{q, 4}\right]\right)\right) .
$$

It implies that $\mu\left(X\left(\left[y_{q, 0}, y_{q, 5}\right]\right)-X\left(\left[y_{q, 1}, y_{q, 4}\right]\right)\right)=0$. Since $\mu$ is a full support measure, we have that $X\left(\left[y_{q, 0}, y_{q, 5}\right]\right)-X\left(\left[y_{q, 1}, y_{q, 4}\right]\right)$ has empty interior. However, we see that the nonempty subset $X\left(\left[y_{q, 0}, y_{q, 1}\right]\right)-\left\{y_{q, 0}, y_{q, 1}\right\}$, which is contained in $X\left(\left[y_{q, 0}, y_{q, 5}\right]\right)-X\left(\left[y_{q, 1}, y_{q, 4}\right]\right)$, is open in $X$ by (a) of Lemma 3.6. This is a contradiction. Thus we complete the proof.

\section{ACKNOWLEDGMENTS}

The first author was supported by the National Natural Science Foundation of China (No. 10801103) and by the Natural Sciences Fund for Colleges and Universities in Jiangsu Province (No. 08KJB110010). The third author was supported in part by the National Natural Science Foundation of China (No. 10871142).

We are grateful to the referee for valuable suggestions.

\section{REFERENCES}

[1] J. Auslander, Minimal flows and their extensions, North-Holland Mathematics Studies, 153, North-Holland, 1988. MR956049 (89m:54050)

[2] B. H. Bowditch, Hausdorff dimension and dendritic limit sets, Math. Ann. 332 (2005), 667676. MR 2181766 (2006k:57043)

[3] Y. N. Minsky, On rigidity, limit sets, and ends of hyperbolic 3-manifolds, J. Amer. Math. Soc. 7 (1994), 539-588. MR1257060 (94m:57029) 
[4] J. H. Mai, E. H. Shi, The nonexistence of expansive commutative group actions on Peano continua having free dendrites, Topology Appl. 155 (2007), 33-38. MR2363562(2008j:37034)

[5] S. B. Nadler, Jr., Continuum Theory: An Introduction, Marcel Dekker, 1992. MR1192552 (93m:54002)

[6] A. L. T. Paterson, Amenability, American Mathematical Society, Providence, RI, 1988. MR961261 (90e:43001)

[7] J. G. Ratcliffe, Foundations of hyperbolic manifolds, Grad. Texts Math., 149, Springer, 2006. MR2249478 (2007d:57029)

[8] E. H. Shi, B. Y. Sun, Fixed point properties of nilpotent group actions on 1-arcwise connected continua, Proc. Amer. Math. Soc. 137 (2009), 771-775. MR2448600

Department of Mathematics, Soochow University, No. 1 Shizi Street, Suzhou 215006 , People's Republic of China

E-mail address: ehshi@yahoo.cn

Department of Mathematics, Soochow University, No. 1 Shizi Street, Suzhou 215006 , People's Republic of China

E-mail address: wangsuhuasz@yahoo.com.cn

Department of Mathematics, Soochow University, No. 1 Shizi Street, Suzhou 215006 , People's Republic of China

E-mail address: zhoulizhen@suda.edu.cn 